\title{
A Note on the correspondence between Qubit Quantum Operations and Special Relativity
}

\author{
Pablo Arrighi ${ }^{1, *}$ and Christophe Patricot ${ }^{2}$,** \\ ${ }^{1}$ Computer Laboratory, University of Cambridge, \\ 15 JJ Thomson Avenue, Cambridge CB3 OFD, U.K. \\ 2 DAMTP, University of Cambridge, Centre for Mathematical Sciences, \\ Wilberforce Road, Cambridge CB3 OWA, U.K.
}

\begin{abstract}
We exploit a well-known isomorphism between complex hermitian $2 \times 2$ matrices and $\mathbb{R}^{4}$, which yields a convenient real vector representation of qubit states. Because these do not need to be normalized we find that they map onto a Minkowskian future cone in $\mathbb{E}^{1,3}$, whose vertical cross-sections are nothing but Bloch spheres. Pure states are represented by light-like vectors, unitary operations correspond to special orthogonal transforms about the axis of the cone, positive operations correspond to pure Lorentz boosts. We formalize the equivalence between the generalized measurement formalism on qubit states and the Lorentz transformations of special relativity, or more precisely elements of the restricted Lorentz group together with future-directed null boosts. The note ends with a discussion of the equivalence and some of its possible consequences.
\end{abstract}

PACS numbers: 03.30, 03.65, 03.67

Keywords: Cones, Bloch sphere, Spinors, Clifford algebras, Generalized measurements

\section{PRELIMINARIES AND GEOMETRICAL SETTING}

This paper may be viewed as a complement to conal representations of quantum states [1]. This section reproduces some of the material in a concise manner, in an attempt to make the presentation self-contained.

The state of a two dimensional quantum system (a qubit) is an element of $\mathrm{Herm}_{2}^{+}(\mathbb{C})$ the set of $2 \times 2$ positive complex matrices [2]. Traditionally one tends to consider normalized states only, i.e. unit trace $\operatorname{Herm}_{2}^{+}(\mathbb{C})$ matrices (density matrices). Yet relaxing this condition has a clear physical meaning and we will often do so in this note. The most general evolution a qubit state may undergo is a generalized measurement (the only extra feature Kraus operators allow is the possibility to ignore one's knowledge of some measurement outcomes). These are described by a finite set $\left\{M_{m}\right\}$ of $2 \times 2$ complex matrices satisfying $\sum_{m} M_{m}^{\dagger} M_{m}=\mathbb{I}$. If we let $E_{m}=M_{m}^{\dagger} M_{m}$ we have that $\sum_{m} E_{m}=\mathbb{I}, E_{m} \in$ $\operatorname{Herm}_{2}^{+}(\mathbb{C})$ and $M_{m}=U_{m} \sqrt{E_{m}}$ using the polar decomposition. Applied upon a density matrix $\rho$, the generalized measurement $\left\{M_{m}\right\}$ yields outcome $m$ with probability $p(m)=\operatorname{Tr}\left(E_{m} \rho\right)$, in which case the post-measurement state is given by $\rho_{m}^{\prime}=\left(1 / \operatorname{Tr}\left(E_{m} \rho\right)\right)\left(M_{m} \rho M_{m}^{\dagger}\right)$. We shall call $\rho_{m}=M_{m} \rho M_{m}^{\dagger} \in \operatorname{Herm}_{2}^{+}(\mathbb{C})$ the unrescaled post-measurement state. Note that the generalized measurement formalism can be viewed as arising when the system is first coupled to an ancilla (through a unitary operation), which then gets measured projectively and discarded. This paper takes the more axiomatic view on

*Electronic address: pja35@cam.ac.uk

** Electronic address: cep29@cam.ac.uk generalized quantum measurements.

Let $\left\{\sigma_{\mu}\right\}_{\mu=0 \ldots 3}$ designate the set of the Pauli matrices $\mathbb{I}, \mathbf{X}, \mathbf{Y}$ and $\mathbf{Z}$. These form a Hilbert-Schmidt orthogonal basis of $2 \times 2$ hermitian matrices, that is $\forall \mu, \nu \operatorname{Tr}\left(\sigma_{\mu} \sigma_{\nu}\right)=2 \delta_{\mu \nu}$ with $\delta$ the Kronecker delta. Thus any matrix $A \in \operatorname{Herm}_{2}(\mathbb{C})$ decomposes on this basis as

$$
A=(1 / 2)\left(\operatorname{Tr}(A) \mathbb{I}+\operatorname{Tr}\left(A \sigma_{i}\right) \sigma_{i}\right)=(1 / 2) \operatorname{Tr}\left(A \sigma_{\mu}\right) \sigma_{\mu}
$$

Notice that throughout this article latin indices run from 1 to 3 , greek indices from 0 to 3 , and repeated indices are summed unless specified. Letting $\underline{A}_{\mu}=\operatorname{Tr}\left(A \sigma_{\mu}\right)$, we shall call $\underline{A}$ the vector $\left(\underline{A}_{\mu}\right) \in \mathbb{R}^{4}$ while $\vec{A}=\left(\underline{A}_{i}\right)$ will designate the restricted vector in $\mathbb{R}^{3}$. Note that the coordinate map

$$
\begin{aligned}
\phi: \operatorname{Herm}_{2}(\mathbb{C}) & \rightarrow \mathbb{R}^{4} \\
A & \mapsto \underline{A}
\end{aligned}
$$

is an isometric isomorphism, in the sense that

$$
\operatorname{Tr}(A B)=\frac{1}{2} \underline{A} \cdot \underline{B} \equiv \frac{1}{2} \underline{A}_{\mu} \underline{B}_{\mu}
$$

Lemma 1 The cone of positive hermitian matrices $\operatorname{Herm}_{2}^{+}(\mathbb{C})$ is isomorphic to the following cone of revolution in $\mathbb{R}^{4}$ :

$$
\Gamma=\left\{\left(\lambda_{\mu}\right) \in \mathbb{R}^{4} / \lambda_{0}^{2}-\sum_{i=1}^{3} \lambda_{i}^{2} \geq 0, \lambda_{0} \geq 0\right\}
$$

Generalized pure states lie on the boundary of $\Gamma$.

Proof: Let $A \in \operatorname{Herm}_{2}(\mathbb{C})$. Its eigenvalues are given by $\lambda_{ \pm}=\frac{1}{2}\left(\underline{A}_{0} \pm \sqrt{\underline{A}_{i} \underline{A}_{i}}\right) . A$ is positive if and only if $\lambda_{+} \lambda_{-} \geq 0$ and $\lambda_{+}+\lambda_{-} \geq 0$. This is equivalent to:

$$
\eta_{\mu \nu} \underline{A}_{\mu} \underline{A}_{\nu} \geq 0 \quad \text { and } \quad \underline{A}_{0} \geq 0
$$


with $\eta_{\mu \nu}=\operatorname{Diag}(1,-1,-1,-1)$. Moreover $A$ is generalized pure when one of its eigenvalues is zero, which is equivalent to $\eta_{\mu \nu} \underline{A}_{\mu} \underline{A}_{\nu}=0$.

Thus the generalized (not necessarily normalized) density matrices of a qubit cover the whole Minkowskian future-light-cone in $\mathbb{E}^{1,3}$. Taking a vertical cross-section of the cone is equivalent to fixing the trace $\underline{A}_{0}$ of the density matrix, which might be thought of physically as the overall probability of occurrence for the state. By doing so we are left with only the spin degrees of freedom along $\mathbf{X}, \mathbf{Y}$ and $\mathbf{Z}$, and therefore each vertical cross-section is a Bloch sphere with radius $\underline{A}_{0}$.

Where the use of Clifford algebras is encountered such a representation is not totally uncommon. We think, for instance, of the community of geometric algebras [3]. Furthermore $\phi^{-1}$ is precisely the isomorphism used to define Dirac spinors [4] in Quantum Field Theories. For $n$-dimensional extensions of the representation we refer the reader to [1] and [5].

We now consider the map $\psi$ from $2 \times 2$ complex matrices to endomorphisms of $\mathbb{R}^{4}$ given by:

$$
\psi: A \mapsto \phi \circ A d_{A} \circ \phi^{-1}
$$

i.e. $\psi(A)$ is the $4 \times 4$ real matrix taking a vector $\rho$ into $A \rho A^{\dagger}$. Notice that $\psi(A B)=\psi(A) \psi(B)$. Amongst the standard results [2] we have that $\psi(U)$, with $U$ unitary, is a special orthogonal transform about the axis of revolution of the cone $\Gamma$. Indeed without loss of generality one can assume $\operatorname{det}(U)=1$, and so the special unitary matrix can be written as:

$$
\begin{aligned}
U=\cos \left(\frac{\theta}{2}\right) \mathbb{I}-i \sin \left(\frac{\theta}{2}\right)\left(\overrightarrow{n_{k}} \sigma_{k}\right) & =e^{-i \frac{\theta}{2} \overrightarrow{n_{k}} \sigma_{k}} \\
\text { and has image: } \quad \psi(U) & =\left(\begin{array}{cc}
1 & 0 \\
0 & R_{\theta}(\vec{n})
\end{array}\right)
\end{aligned}
$$

Here $R_{\theta}(\vec{n})$ denotes the real rotation by an angle $\theta$ around the normalized axis $\vec{n}$ (to happen in the Bloch sphere). Alternatively one may use the expression $\psi(U)_{\mu \nu}=(1 / 2) \operatorname{Tr}\left(U \sigma_{\nu} U^{\dagger} \sigma_{\mu}\right)$. The next formulae are not well-known.

Lemma 2 Let $\sqrt{E_{m}}$ be a matrix in $\operatorname{Herm}_{2}^{+}(\mathbb{C})$, with $\underline{\sqrt{E_{m}}}=\left[\begin{array}{llll}\alpha & \beta & \gamma & \delta\end{array}\right]$, and $E_{m}$ its square, with $\underline{E_{m}}=$ $\overline{[a x y} z]$. Then

$$
\begin{aligned}
\psi\left(\sqrt{E_{m}}\right) & =\frac{1}{4}\left(\begin{array}{cccc}
-X+2 \alpha^{2} & 2 \alpha \beta & 2 \alpha \gamma & 2 \alpha \delta \\
2 \alpha \beta & X+2 \beta^{2} & 2 \beta \gamma & 2 \beta \delta \\
2 \alpha \gamma & 2 \beta \gamma & X+2 \gamma^{2} & 2 \gamma \delta \\
2 \alpha \delta & 2 \beta \delta & 2 \gamma \delta & X+2 \delta^{2}
\end{array}\right) \\
= & \frac{1}{4}\left(\begin{array}{cccc}
2 a & 2 x & 2 y & 2 z \\
2 x & X+\frac{4 x^{2}}{2 a+X} & \frac{4 x y}{2 a+X} & \frac{4 x z}{2 a+X} \\
2 y & \frac{4 x y}{2 a+X} & X+\frac{4 y^{2}}{2 a+X} & \frac{4 y z}{2 a+X} \\
2 z & \frac{4 x z}{2 a+X} & \frac{4 y z}{2 a+X} & X+\frac{4 z^{2}}{2 a+X}
\end{array}\right)
\end{aligned}
$$

with $X=\alpha^{2}-\beta^{2}-\gamma^{2}-\delta^{2}=2 \sqrt{a^{2}-x^{2}-y^{2}-z^{2}}$.
Proof: $\psi\left(\sqrt{E_{m}}\right)$ can be computed in terms of $\underline{\sqrt{E_{m}}}$ using the following simple formula:

$$
\psi\left(\sqrt{E_{m}}\right)_{\mu \mu^{\prime}}=(1 / 2) \underline{\sqrt{E_{m}}} \nu{\underline{\sqrt{E_{m}}}}_{\nu^{\prime}} \operatorname{Tr}\left(\sigma_{\nu} \sigma_{\mu^{\prime}} \sigma_{\nu^{\prime}} \sigma_{\mu}\right)
$$

This method requires lengthy calculations, subtler approaches are discussed in $[1]$. Now let $\underline{\iota}=\left[\begin{array}{llll}1 & 0 & 0 & 0\end{array}\right]=$ $(1 / 2) \phi(\mathbb{I})$ and observe that

$$
\begin{aligned}
\psi\left(\sqrt{E_{m}}\right) \underline{\iota} & \equiv \phi \circ A d_{\sqrt{E_{m}}} \circ \phi^{-1} \underline{\iota} \\
& =(1 / 2) \phi\left(\sqrt{E_{m}} \mathbb{I} \sqrt{E_{m}}\right) \equiv(1 / 2) \underline{E_{m}}
\end{aligned}
$$

In other words, $(1 / 2) \underline{E_{m}}$ has as components the first column of $\psi\left(\sqrt{E_{m}}\right)$. Thus we can now proceed to the substitutions which yield the second form of $\psi\left(\sqrt{E_{m}}\right)$. Finally the $X$ relation stems from:

$$
\begin{aligned}
\eta_{\mu \nu} \underline{\sqrt{E_{m}}} \mu \underline{\sqrt{E_{m}}} \nu & =4 \operatorname{det}\left(\sqrt{E_{m}}\right) \\
& =4 \sqrt{\operatorname{det}\left(E_{m}\right)}=2 \sqrt{\eta_{\mu \nu} \underline{E_{m}} \mu \frac{E_{m}}{\nu}}
\end{aligned}
$$

\section{QUANTUM OPERATIONS AS LORENTZ TRANSFORMS AND VICE-VERSA}

We begin by showing that elements of a generalized measurement act on a qubit either as rescaled restricted Lorentz transformations or as rescaled future-directed null boosts. Then we show that the reverse is also true. Remember that a Lorentz transform $L \equiv L_{\nu}^{\mu}$ is called restricted if it is proper ( $\operatorname{det} L=1$ ) and orthochronous $\left(L_{0}^{0}>0\right)$. We will show that such an $L$ decomposes uniquely into the product of a proper spatial rotation and a pure (timelike future-directed velocity) boost. We like to think of null velocity boosts as limiting cases of restricted boosts, or effectively as elements of the topological boundary of the restricted Lorentz group, but they need to be rescaled to yield a finite linear transform. We shall call these (rescaled) future-directed null boosts. They are singular transforms. It turns out the rescaling introduced defines a natural unifying way of thinking about Lorentz transforms and null boosts.

If $\underline{E_{m}}=[a, x, y, z]$ corresponds to one particular measurement element $E_{m}=M_{m}^{\dagger} M_{m}$, we shall call $\underline{V_{m}}$ the vector of coordinates $\left(\underline{V}_{m} \mu\right)=\left(\frac{1}{2} \eta_{\mu \nu} \underline{E}_{m}\right)$, i.e. $\frac{V_{m}}{a}=[a / 2,-x / 2,-y / 2,-z / 2]$. Then $\bar{v}_{m}=$ $2 V_{m} / \vec{a}$ is the corresponding normalized vector and $\overrightarrow{v_{m}}=$ $[\overline{-x} / a,-y / a,-z / a]$ can be thought of as a three vector velocity, whose norm is defined as usual: $v_{m}=$ $\left(\overrightarrow{v_{m}} \cdot \overrightarrow{v_{m}}\right)^{1 / 2}$.

Proposition 1 Let $\left\{M_{m}\right\}=\left\{U_{m} \sqrt{E_{m}}\right\}$ be a generalized measurement on a qubit, with $U_{m}$ unitary and $\sqrt{E_{m}}$ positive. Then for all $m$ such that $E_{m}$ is not projective, we have:

$$
\psi\left(M_{m}\right)=\sqrt{\eta_{\mu \nu} \underline{V_{m}} \mu \underline{V_{m}} \nu} R_{m} L\left(\underline{v_{m}}\right)
$$


where $R_{m}=\psi\left(U_{m}\right)$ is a proper rotation about the axis of the cone and $L\left(\underline{v_{m}}\right)$ is a pure restricted Lorentz boost of normalized velocity $v_{m}$. Thus $\psi\left(M_{m}\right)$ is a restricted Lorentz transform up to a (strictly positive) scalar. Similarly, if $E_{m}$ is projective, $\psi\left(M_{m}\right)=(a / 2) R_{m} L\left(v_{m}\right)$, where $L\left(\underline{v}_{m}\right)$ is a rescaled pure future-directed null boost of null velocity $\underline{v_{m}}$.

Proof: First recall that $\psi\left(M_{m}\right)=\psi\left(U_{m}\right) \psi\left(\sqrt{E_{m}}\right)$, and by (2), $\psi\left(U_{m}\right)$ is a special orthogonal transformation about the axis of the cone, so a restricted Lorentz transform. Suppose $E_{m}$ (hence $v_{m}$ ) timelike future-directed. Letting $\gamma \equiv 2 a / \bar{X}$ in (3) and using the definition of $\overrightarrow{v_{m}}$, we get:

$$
\frac{4}{X} \psi\left(\sqrt{E_{m}}\right)=\left(\begin{array}{cc}
\gamma & -\gamma{\overrightarrow{v_{m}}}^{T} \\
-\gamma \overrightarrow{v_{m}} & \mathbb{I}+\frac{\gamma^{2}}{1+\gamma} \overrightarrow{v_{m}}{\overrightarrow{v_{m}}}^{T}
\end{array}\right) \equiv L\left(\underline{v_{m}}\right)
$$

As $\gamma=1 / \sqrt{1-v_{m}^{2}}, L\left(\underline{v_{m}}\right)$ is precisely a pure Lorentz boost of velocity $\underline{v}_{m}$ (see [4] for example). Since $\underline{v_{m}}$ is timelike future-directed, $\psi\left(M_{m}\right)$ is a restricted Lorentz transform up to the factor $X / 4=(1 / 2) \sqrt{\eta_{\mu \nu} \underline{E}_{m} \mu \underline{E}_{m}}=$ $\sqrt{\eta_{\mu \nu} \underline{V}_{m} \frac{V_{m}}{\nu}}$.

Now, when $E_{m}$ is null ( $E_{m}$ projective) this factor vanishes and $\gamma$ becomes infinite. Nevertheless one can write (3) for $X=0$ as

$$
\frac{2}{a} \psi\left(\sqrt{E_{m}}\right)=\left(\begin{array}{cc}
1 & -{\overrightarrow{v_{m}}}^{T} \\
-\overrightarrow{v_{m}} & \overrightarrow{v_{m}}{\overrightarrow{v_{m}}}^{T}
\end{array}\right)
$$

We can see that this is in fact a pure null boost rescaled by a factor $\gamma^{-1}$. Indeed, when $v_{m} \rightarrow 1$ the right-handside of (7) becomes

$$
L^{\text {null }}\left(\underline{\underline{v_{m}}}\right) \sim \gamma\left(\begin{array}{cc}
1 & -{\overrightarrow{v_{m}}}^{T} \\
-\overrightarrow{v_{m}} & \overrightarrow{v_{m}}{\overrightarrow{v_{m}}}^{T}
\end{array}\right)
$$

and since $\frac{a}{2}=\gamma \sqrt{\eta_{\mu \nu} \underline{V}_{m} \underline{V}_{m}}$, we precisely get

$$
\psi\left(\sqrt{E_{m}}\right) \sim \sqrt{\eta_{\mu \nu} \underline{V}_{m} \mu \underline{V}_{m}} L^{\text {null }}\left(\underline{v_{m}}\right)
$$

Here the Minkowski product vanishes and the unrescaled pure null velocity boost is infinite. Nevertheless rescaling $L^{\text {null }}\left(\underline{v_{m}}\right)$ by the factor $\gamma^{-1}$ yields the right-hand-side of (8); thus $\psi\left(\sqrt{E_{m}}\right)$ indeed corresponds to a rescaled pure null boost, which of course is not an element of the Lorentz group.

As we said previously the natural rescaling by the Minkowski product precisely corresponds to an appropriate rescaling of generalized Lorentz transforms bringing null boosts to finite linear maps. Formally the essence of this Proposition can be thought of as a consequence of the Alexandrov-Zeeman theorems relating the causality group (Lorentz group and dilatations) to the Minkowskian causal structure, though this approach would not cover null velocity boosts. Note that the rescaled pure null velocity boosts (right-hand-side of [8]) are in fact proportional to projections on the null four vectors $E_{m}$.

Maybe the reader wonders here why the Lorentz pure boosts corresponding to positive measurement elements $E_{m}$ are parametrized by $\underline{v}_{m}$ and not $\underline{E_{m}}$. However since $E_{m}$ is an operator acting on states and not a state, $E_{m}$ is better thought of as a co-vector, or element of the $\overline{d u a l}$ space, in the same way as momenta are dual to positions in usual Special Relativity. The (contravariant) vector corresponding to $\underline{E_{m}}$ is precisely $2 \underline{V_{m}}$, thus in the space of states, and not operators, $E_{m}$ is represented by $2 V_{m}$. The factor of two was introduced merely for convenience.

The following relations suggest the Minkowski product of the state vector of a qubit is an important quantum information theoretical quantity:

Proposition 2 Let $\left\{M_{m}\right\}$ be a generalized measurement, $\underline{\rho}$ a state vector and $\psi\left(M_{m}\right) \underline{\rho} \equiv \rho_{m}$ the unrescaled post-measurement state vector if outcome $m$ occurs. We have:

$$
\begin{aligned}
\eta_{\mu \nu} \underline{\rho}_{m} \underline{\rho}_{m} & =\eta_{\mu \nu} \underline{V}_{m} \underline{V}_{\mu}{\underline{V_{m}}}_{\nu} \eta_{\mu^{\prime} \nu^{\prime}} \underline{\rho}_{\mu^{\prime}} \underline{\rho}_{\nu^{\prime}} \\
\underline{\rho}_{m} & =\eta_{\mu \nu} \underline{V}_{m} \underline{\rho}_{\nu} \\
\eta_{\mu \nu} \underline{\rho}_{\mu} \underline{\rho}_{\nu} & =2\left([\operatorname{Tr}(\rho)]^{2}-\operatorname{Tr}\left(\rho^{2}\right)\right)
\end{aligned}
$$

Proof: We make use of the previous proposition. Equation (6) implies

$\eta_{\mu \nu} \underline{\rho}_{\mu} \underline{\rho}_{\nu}=\eta_{\mu \nu} \underline{V}_{m} \underline{V}_{m} \underline{V}_{\nu} \eta_{\mu^{\prime} \nu^{\prime}}\left(R_{m} L\left(\underline{v_{m}}\right) \underline{\rho}\right)_{\mu^{\prime}}\left(R_{m} L\left(\underline{v_{m}}\right) \underline{\rho}\right)_{\nu^{\prime}}$

and (9) follows since $R_{m} L\left(v_{m}\right)$ is a Lorentz transform. This relation remains true of course when $V_{m}$ is light-like ( $E_{m}$ projective), since so is $\rho_{m}$. (Purity relations [1]). For the second equation note that $\underline{\rho}_{m}=\operatorname{Tr}\left(E_{m} \rho\right)=$ $(1 / 2) E_{m} . \rho$, where the isometry (1) was applied. Introducing the definition of $\underline{V_{m}}$ in this last equation yields the required result.

Equation (11) can be shown explicitly using the components of $\rho$ and $\rho^{2}$, but it seems more interesting to use our isomorphism $\phi: \rho \rightarrow \underline{\rho}$. Consider the linear map on $\mathbb{E}^{1,3}, \Lambda:\left(\underline{\rho}_{\mu}\right) \rightarrow\left(\eta_{\nu \mu} \underline{\rho}_{\nu}\right)$ (musical isomorphism). Then $\widetilde{\Lambda}: \rho \rightarrow \phi^{-1} \circ \Lambda \circ \phi(\rho)$ is a linear map on $\operatorname{Herm}_{2}(\mathbb{C})$. One finds easily $\widetilde{\Lambda}(\rho)=(\operatorname{Tr} \rho) \mathbb{I}-\rho$. Using the fact that $\phi$ is an isometry (11), we get

$$
\begin{aligned}
\eta_{\mu \nu} \underline{\rho}_{\mu} \underline{\rho}_{\nu} & \equiv(\Lambda \underline{\rho}) \cdot \underline{\rho}=2 \operatorname{Tr}(\widetilde{\Lambda}(\rho) \rho) \\
& =2\left([\operatorname{Tr} \rho]^{2}-\operatorname{Tr}\left(\rho^{2}\right)\right)
\end{aligned}
$$

It seems interesting that this quantity, invariant under Lorentz transforms on the state vector $\rho$, in fact measures the mixedness of qubit states: recall that a density matrix $\rho$ is pure if and only if $\operatorname{Tr}\left(\rho^{2}\right)=(\operatorname{Tr} \rho)^{2}$. Not only is purity preserved under a formal Lorentz boost, so is this notion of mixedness. Moreover this quantity 
maps according to the simple relation (9) under a generalized measurement. Note that since $\eta_{\mu \nu} \underline{V}_{m} \mu \underline{V}_{m} \leq 1$, the mixedness always decreases given a measurement outcome. But (9) and (10) suggest much more: the mixedness of post-measurement states and their probabilities are invariant if both the initial vector $\rho$ and the measurement vectors $V_{m}$ are Lorentz transformed. However, the set of transformed measurement vectors does not sum to the identity, and it is unclear how to interpret it as a quantum measurement. In section III we will discuss the way a boosted observer perceives measurement probabilities, but without using the approach equation (10) might suggest. We now show that any Lorentz transformation can be thought of as an element of a generalized measurement up to scale.

Proposition 3 Let $L$ a restricted Lorentz transform or a rescaled future-directed null boost of $\mathbb{E}^{1,3}$. L decomposes as $L=R L(\underline{v})$ where $R$ is a proper Lorentz rotation and $L(\underline{v})$ a pure velocity boost, rescaled when $\underline{v}$ is null. Then there exits a particular element of a measurement scheme $\left\{M_{m}\right\}, M_{1}$ say, such that for any qubit $\rho$,

$$
L \underline{\rho} \propto \psi\left(M_{1}\right) \underline{\rho}
$$

Thus the effect of a Lorentz boost on a qubit can essentially be viewed as applying a particular measurement element whose outcome occurs. More precisely there exits a family of such possible measurement elements $M(\lambda)=U \sqrt{E(\lambda)}$ defined by $U=U(R)$ as in (2) and $\sqrt{E(\lambda)}$ satisfying the following:

If $L=R L(\underline{v})$ is a restricted Lorentz transform:

$$
\begin{aligned}
& \underline{\sqrt{E(\lambda)}}=\left(1+\sqrt{1-v^{2}}\right)^{-1 / 2}\left[\lambda\left(1+\sqrt{1-v^{2}}\right),-\lambda \vec{v}\right] \\
& \text { with } 0<\lambda \leq \sqrt{\frac{2}{1+v}}
\end{aligned}
$$

while if $L=R L(\underline{v})$ is a rescaled future-directed null boost:

$$
\underline{\sqrt{E(\lambda)}}=[\lambda,-\lambda \vec{v}] \text { with } 0<\lambda \leq 1 .
$$

Proof: For completeness we first show the decomposition of restricted Lorentz transforms $L$ into $L=R L(\underline{v})$ as above. This relies on the well-known spinor representation of the restricted Lorentz group, or the two-to-one group homomorphism between unimodular $2 \times 2$ complex matrices and restricted Lorentz transforms (see [4] for example):

$$
\begin{aligned}
\psi: S L(2, \mathbb{C}) & \rightarrow S O(1,3)^{+} \\
A & \mapsto \psi(A) \equiv \phi \circ A d_{A} \circ \phi^{-1}
\end{aligned}
$$

Indeed as $A d_{A}$ preserves the determinant and $\phi$ is such that for all $\rho \in \operatorname{Herm}_{2}(\mathbb{C}), \operatorname{det} \rho=(1 / 4) \eta_{\mu \nu} \underline{\rho}_{\mu} \underline{\rho}_{\nu}, \psi(A)$ preserves the Minkowski product. The fact that $\psi(A) \in$ $S O(1,3)^{+}$and that $\psi$ is two-to-one and onto can be checked explicitly. Let $L$ any restricted Lorentz transform. There exits a unique $A \in S L(2, \mathbb{C})$ such that $\psi( \pm A)=L$. Polar decompose $A$ into $A=U|A|$ with $U$ unitary and $|A|$ positive. ( $U$ is in fact special unitary and $|A|$ positive definite since $\operatorname{det} A=1$, and by unicity of the polar decomposition for $A$ non-singular, $-A=(-U)|A|)$. Applying Proposition 11 to $|A|$ with $\operatorname{det}|A|^{2}=1, \psi(|A|)$ is a pure restricted Lorentz boost, thus $L=\psi(U) \psi(|A|)$ provides a decomposition. Since $\psi(U)=\psi(-U)$, this decomposition is unique.

Thus given $L=R L(\underline{v})$, with $R$ a proper rotation and $L(\underline{v})$ a pure boost of future-directed timelike velocity $\underline{v}=[1, \vec{v}]$, we use Proposition 11 to find $M=U \sqrt{E}$ such $\psi(M) \propto L . \quad U=U(R)$ is given by (2) and we choose $\underline{E}=[1,-\vec{v}]$.

We then have to find $\lambda>0$ such that $\lambda M$ can be part of a measurement scheme. This is equivalent to $\lambda^{2} M^{\dagger} M$ positive (satisfied) and $\mathbb{I}-\lambda^{2} M^{\dagger} M$ positive too. ( $\lambda M$ and $-\lambda M$ are equivalent in terms of measurement elements). With $\lambda M=U \sqrt{E(\lambda)}$, we have

$$
\underline{E(\lambda)}=\left[\lambda^{2},-\lambda^{2} \vec{v}\right]
$$

from which we find $\underline{\sqrt{E(\lambda)}}$ using (4):

$$
\underline{\sqrt{E(\lambda)}}=\left(1+\sqrt{1-v^{2}}\right)^{-1 / 2}\left[\lambda\left(1+\sqrt{1-v^{2}}\right),-\lambda \vec{v}\right] .
$$

Then requiring $\mathbb{I}-E(\lambda)$ positive is equivalent to $(\lambda>0)$ :

$$
\lambda \leq \sqrt{\frac{2}{1+v}}
$$

Applying Proposition 1 we get:

$$
\psi(M(\lambda))=\frac{\lambda^{2}}{2} \sqrt{1-v^{2}} R L(\underline{v})
$$

Thus for such $\lambda$ the measurement elements $M(\lambda)=$ $U \sqrt{E(\lambda)}$ are all possible measurements whose occurrence is equivalent up to a factor to the restricted Lorentz boost $L=R L(\underline{v})$.

Now let $L$ a rescaled future-directed null boost. As we have shown, any restricted Lorentz transform can be decomposed into a product of a proper rotation and a boost of time-like future-directed velocity. Future-directed null boosts are just limits of these, and thus the rescaled null boosts $L$ may be assumed to be the product of a rotation $R$ and a rescaled null pure boost $L(\underline{v})$ of type (8). The rotation can be dealt with as in the previous case. Defining $\underline{E}=[1,-\vec{v}]$ null future-directed, we have $L(\underline{v}) \propto \psi\left(\phi^{-1}(\underline{\sqrt{E}})\right)$. Then again we consider $E(\lambda)=\lambda^{2} \underline{E}(\lambda>0)$ such that $\mathbb{I}-E(\lambda)$ is positive. This is equivalent to $0<\lambda \leq 1$, and using (4) we have

$$
\underline{\sqrt{E(\lambda)}}=[\lambda,-\lambda \vec{v}]
$$

which gives $\psi(\sqrt{M(\lambda)})=\left(\lambda^{2} / 2\right) R L(\underline{v})$.

Note that the scaling factor is always less than 1 , indeed less than $\sqrt{(1-v) /(1+v)}$ in the restricted case, and $1 / 2$ in the null case. 
Overall we have shown that elements of generalized measurements on a qubit are equivalent to rescaled restricted or null Lorentz transforms. Projective measurement elements are future-directed null boosts, while mixed ones correspond to restricted Lorentz boosts. One can of course think of these linear transforms as elements or limits of elements of the causality group of $\mathbb{E}^{1,3}$.

\section{DISCUSSION}

The following is a somewhat original discussion of Propositions 11 to 3] Our formalism and its consequences suggest that qubit states may be viewed as spatio-temporal objects, or indeed as four-vectors of a Minkowski space-time. This differs only slightly from the notion of spin as a spatial polarization direction, and thus may apply to 2 dimensional quantum systems whose degrees of freedom can be thought of as spacelike. We shall adopt this point of view from now, i.e consider naively qubits as four-vectors, and analyse the physical implications.

Let us begin by merely rephrasing the content of the correspondence that was established in section [I Suppose Alice proceeds to a generalized measurement $\left\{M_{m}\right\}=$ $\left\{U_{m} \sqrt{E_{m}}\right\}, \sum_{m} M_{m}^{\dagger} M_{m}=\mathbb{I}$ on a qubit density matrix $\rho$ ( $\rho$ is unit trace). With probability $p(m)=\operatorname{Tr}\left(E_{m} \rho\right.$ ) this will yield her a (non-normalized) post-measurement state $\rho_{m}=M_{m} \rho M_{m}^{\dagger}$. This rather common situation turns out to be equivalent, according to Proposition 1 to the following less usual scenario:

Scenario 1: Suppose Alice is standing at the origin of an inertial frame of Minkowski spacetime, contemplating the four-vector $\rho$. Say she gives herself a set of rotations $\left\{R_{m}\right\}$ and four-vectors $\left\{\underline{V_{m}}\right\}$ such that $\sum_{m} \underline{V_{m}}=$ $\left[\begin{array}{llll}1 & 0 & 0 & 0\end{array}\right]$. Now, with probability $p(m)=\eta_{\mu \nu} \underline{V_{m}} \mu \underline{\rho}_{\nu}$ she chooses to Lorentz boost herself up to velocity vector $\frac{v_{m}}{R_{m}}=\underline{V_{m}} / \underline{V}_{m_{0}}$, to rotate the resulting space-frame by $\overline{R_{m}}$ and to rescale her coordinates by a factor of $\sqrt{\eta_{\mu \nu} \underline{V}_{m} \underline{V}_{m}} \nu$ (we are assuming $E_{m}$ is not projective). She then looks back upon her object of contemplation and sees $\rho_{m}$, the unrescaled post-measurement state. The case with $E_{m}$ projective is the limit of the previous one when the boost vector $\underline{v_{m}}$ becomes null, and the rescaling yields finiteness of the corresponding linear transform.

Therefore a quantum measurement can be thought of, up to scale, as the observer taking a Lorentz boost relative to his or her qubit. Notice that applying a second quantum measurement $\left\{N_{n}\right\}$ similarly corresponds to the observer taking a second (successive) Lorentz transformation at random amongst $\left\{L_{n}\right\}$, say. Thus qubit quantum mechanics can easily be axiomatized within the mathematics of special relativity, and pure measurement elements go hand-in-hand with future-directed null boosts.

Difficulties are prompt to arise when one seeks to equate a measurement interaction, in which the qubit is physically acted upon, with a (somewhat passive) coordinate transformation in Minkowski space-time: indeed the latter is purely kinematical, thus reversible, whereas the former usually implies a collapse of the state. In the following scenario we dissociate one from the other. In other words we consider special relativity and qubit quantum theory in their most usual fashion, save for the fact that we continue to interpret the spin a a four-vector.

Scenario 2: Suppose Alice at the origin of an inertial frame of Minkowski space, together with a qubit density matrix $\rho$ (unit trace) which we think of as a (normalized) space-time vector $\rho$. If we consider the point of view of Bob as he passes by in an inertial frame, this suggests that Bob sees a boosted version of $\rho$, i.e. a state $\Lambda \rho$. This seemingly innocuous point raises an important issue however: $\Lambda$ is not restricted to Bloch sphere rotations, and thus may indeed not correspond to a unitary operation. To understand its effect upon $\rho$ we must refer to Proposition 14 acts, up to a factor, as a measurement element $M_{1}$ whose outcome always happens, even though $\operatorname{Tr}\left(M_{1} \rho M_{1}^{\dagger}\right) \neq 1$. Thus $\left\{M_{1}\right\}$ can be thought of as a non trace-preserving quantum operation $\left(M_{1} M_{1}^{\dagger} \neq \mathbb{I}\right)$ which systematically occurs. We shall let $\underline{\rho^{B o b}} \equiv \Lambda \underline{\rho} \propto \underline{M_{1} \rho M_{1}^{\dagger}}$ and proceed to reassure the reader: such a phenomenon would not violate the principle of relativity. Bob does not make happen a non trace-preserving quantum operation on the qubit. The laws of quantum mechanics remain exactly the same in every inertial frame: only the change of observers, or more precisely the way a boosted observer perceives a non-boosted state, is a non-orthodox quantum operation. If Bob were then to decelerate down to the speed of Alice, his mathematical description of the qubit would return to be $\rho$ again.

Now suppose Alice measures $\rho$ under a generalized measurement $\left\{N_{n}\right\}$. The probability associated with the transition from $\rho$ to $\rho_{n}$ is given by $p(n) \equiv$ $\operatorname{Tr}\left(N_{n}^{\dagger} N_{n} \rho\right) / \operatorname{Tr}(\rho)=\underline{\rho}_{n}$, as usual when $\underline{\rho}$ is normalized. As Bob passes, he sees the initial state $\underline{\rho^{B o b}}=\Lambda \underline{\rho}$, and the post-measurement states $\rho_{n}^{B o b}=\Lambda \overline{\rho_{n}}$. Remember that the probability associated to a state is simply given by the first component of its vector representation. Assuming $\Lambda$ is a pure boost of non-null normalized velocity $\underline{v(\Lambda)}$, we get:

$$
p^{B o b}(n) \equiv \frac{\operatorname{Tr}\left(\rho_{n}^{B o b}\right)}{\operatorname{Tr}\left(\rho^{B o b}\right)}=\frac{\rho_{n}^{B o b} 0}{{\underline{\rho^{B o b}}}_{0}}=\frac{p(n)-\overrightarrow{v(\Lambda)} \cdot \overrightarrow{\rho_{n}}}{1-\overrightarrow{v(\Lambda)} \cdot \vec{\rho}} \geq 0
$$

In other words the probabilities associated with the transitions from $\underline{\rho}$ to $\underline{\rho_{n}}$, in the same way as lengths of objects, are not invariant under a change of observer. Thus if one believes probabilities are absolute quantities independent of notions of space and time, one must abandon trying to interpret the qubit as a 4 -vector.

Otherwise, the notion of probability as a physical quantity needs to be redefined $\left(\sum_{n} p(n)\right.$ is not conserved, as the probability of a state transforms just like the timecomponent of a four vector). The idea is disturbing, and 
certainly worth comparing with the contraction of any spatial object (a ruler, say) under a Lorentz boost. As he passes by Bob will see Alice's $20 \mathrm{~cm}$ ruler shrunk down to $15 \mathrm{~cm}$. But what we now have is that if Alice's quantum ruler has half a chance of being $22 \mathrm{~cm}$ long, and another half chance of measuring $18 \mathrm{~cm}$, it may well turn out that Bob instead perceives a quantum ruler of length $17 \mathrm{~cm}$ with probability a third, and $14 \mathrm{~cm}$ two third of the times.

Allowing the Lorentz boosts $\Lambda$ to act on $\rho$ as on spacetime vectors thus seems a radical departure from Quantum Field Theories in Minkowski space, where the approach is to seek unitary representations of the Poincaré group, i.e. the full Lorentz group together with translations. However, Poincaré invariance (see [ $[\underline{6}$ for example) does not require any given state of a theory to transform unitarily under a change of observer: for any two inertial observers Alice and Bob, it requires the existence, given any state of the theory possibly measured by Alice in her frame, of another state of the theory measured by Bob in his frame, such that the statistics of their measurement outcomes on their respective states be the same. In this sense, the action of a particular Poincaré transform on a state in Quantum Field Theory corresponds to a change of inertial frame: it maps a given solution for an inertial family of observers to another equivalent solution for another family of observers, hence it simply cannot change the measurement statistics. Our second scenario does not involve a change of inertial frame, but just a change of observer. It is true that nonetheless, Alice's non-boosted qubit viewed by a boosted observer Bob, though not necessarily unitarily equivalent to the same non-boosted state viewed by Alice, should be an admissible state of the theory which could be measured by Bob to yield measurement statistics with the usual properties. We are not in this case, since in scenario 2, Bob is not performing a quantum operation on Alice's qubit. Note also that in the formalism developed above, pure states, whether viewed in their inertial frame or not, remain pure.

But if we begin to think of quantum measurement outcome probabilities as not invariant under Lorentz transformations, then the Von Neumann entropy should not be either. On the other hand the invariant quantity $\eta_{\mu \nu} \underline{\rho}_{\mu} \underline{\rho}_{\nu}$ seems a good measure of the mixedness of $\rho$, an idea which is strongly supported by its equivalent form (11). With $I(\underline{\rho})$ proportional to the logarithm of $\eta_{\mu \nu} \underline{\rho}_{\mu} \underline{\rho}_{\nu}$ equation (91) becomes:

$$
I\left(\underline{\rho_{m}}\right)=I\left(\underline{V_{m}}\right)+I(\underline{\rho})
$$

This result is rather interesting as an information conservation law.

The lines of thought suggested in this last section need to be anchored in firmer ground and generalized to higher dimensional quantum systems. Although most of the mathematical results of this paper stem from the exceptional isomorphism between $\operatorname{Herm}_{2}^{+}(\mathbb{C})$ and the future cone of Minkowski space, there is hope to find a special relativistic interpretation to $d$ dimensional systems [1]. This is currently being investigated. More generally the authors feel that the correspondence between qubit quantum operations and special relativity transforms deserves further attention.

\section{ACKNOWLEDGMENTS}

C.E.P would like to thank Gary Gibbons for motivating discussions, EPSRC, the DAMTP, the Cambridge European and Isaac Newton Trusts for financial support. P.J.A would like to thank Anuj Dawar for his patient listening, EPSRC, Marconi, the Cambridge European and Isaac Newton Trusts for financial support.
[1] P.J. Arrighi, C.E. Patricot, The Conal representation of Quantum States and Non Trace-Preserving Quantum Operations, Preprint: quant-ph/0212062

[2] M.A. Nielsen, I.L. Chuang, Quantum Computation and Quantum Information, Cambridge University Press (2000).

[3] T.F. Havel, C.J. Doran, Geometric Algebra in Quantum Information Processing, Preprint: quant-ph/0004031
[4] R.U. Sexl, H.K. Urbantke, Relativity, Groups, Particles, Springer Physics, Springer Wien NewYork, (2001).

[5] P. Zanardi, A Note on Quantum Cloning in $d$ dimensions, Phys. Rev. A 58 (1998) 3484 (Preprint: quant-ph/9804011).

[6] R.M. Wald, Quantum gravity and time reversibility, Phys.Rev.D 51 (1980), pp.2742-2755. 